Voix et Images

voixetimages

\title{
V.4 Coup de barre : sites d'une lecture
}

\section{Paul Chanel Malenfant}

Volume 10, numéro 2, hiver 1985

La barre du jour / La nouvelle barre du jour

URI : https://id.erudit.org/iderudit/013879ar

DOI : https://doi.org/10.7202/013879ar

Aller au sommaire du numéro

\section{Éditeur(s)}

Université du Québec à Montréal

\section{ISSN}

0318-9201 (imprimé)

1705-933X (numérique)

Découvrir la revue

\section{Citer cet article}

Malenfant, P. C. (1985). V.4 Coup de barre : sites d'une lecture. Voix et Images, 10(2), 139-151. https://doi.org/10.7202/013879ar d'utilisation que vous pouvez consulter en ligne.

https://apropos.erudit.org/fr/usagers/politique-dutilisation/ 


\title{
V.4 Coup de barre: sites d'une lecture
}

\author{
par Paul Chanel Malenfant, Université du Québec à Rimouski
}

\section{Premier site}

Automne 1972. Sans doute sommes-nous nombreux, aussi fébriles que curieux, à nous retrouver chaque semaine dans telle classe de l'Université de Montréal. Hélène Cixous fait guichet fermé. À la lettre, elle épelle Bataille, Joyce, Lacan, Genet ... Le cours s'intitule: «Problèmes de la modernité». Mise en scène: signifiant et inconscient du texte, névrose et littérature, formes-sens, femmes et écritures ...

«Etre psychiquement en vie signifie que vous êtes amoureux, en analyse, ou bien en proie à la littérature», écrit maintenant Julia Kristeva en postface à Histoires d'amour. Cet automne-là, je crois bien que nous débordions d'énergie psychique. En plein transfert littéraire. Et par analyste interposé, nous accédions à d'«autres» entrées dans le texte, à des formes transgrèssées de la passion. Est-ce bien la même année que Jean Cohen nous avait fait cette lecture "écartée» de "Cinq heures du soir» de Lorca, que nous avons lu telle analyse des «Chats» de Baudelaire et que Jakobson avait été de passage?

Toujours est-il que quelques mois plus tard, un groupe formé, entre autres de quelques-uns de ces auditeurs assidus des érudites séances de séduction cixousienne, se constituera autour de Nicole Brossard, alors à la direction, avec Roger Soublière, de la BJ. Le projet qui nous rassemble: un numéro spécial de la revue qui proposerait des lectures structurales portées sur des textes-cibles de la poésie québécoise, de Saint-Denys Garneau à Paul-Marie Lapointe, de Rina Lasnier à Claude Gauvreau. Je ne pourrais affirmer qu'il y ait eu une relation de cause à effet ou quelque lien autre que de renforcement ou de coïncidence motivée entre l'atmosphère intellectuelle suscitée par la présence de Cixous et notre «venue à l'écriture» par la lecture privilégiée des formes; certes les questions de la modernité avaient été, à tout le moins, soulevées, et notre attitude quant à la prise (au point) de vue du texte avait été remise en cause. Ainsi, dans la fougue des syntagmes disséqués, la voltige des statistiques prosodiques, la pratique d'une grammaire hautement combinatoire, nous tentions de lire «autrement» nos classiques. $\hat{A}$ bas la «tête thématique», fin des traces biographiques ou nationalistes, des «illusions sémantiques», l'entreprise unanime donne dans le plein des structures, des articulations et des générateurs. «(...) notre acte de lecture indisposera parce que s'y jouant à vide; puisqu'il ne sera question ici que d'y «voir clair» sans pour autant que nous en tirions maintenant les conséquences ${ }^{1}$. . Onze analyses paraîtra au printemps-été 1973. Liviraison 39-40-41.

Volte-face. Je viens de recevoir le numéro 141: Vouloir la fiction @ la modernité, premier numéro qui marque le début de la 20e année d'existence 
de la revue. Que s'est-il donc passé depuis la minutie mécaniste mais enthousiaste de nos lectures structurales, jusqu'à maintenant? Nous avions peut-être été sollicités par les «Problèmes de la modernité», voilà qu'ils se sont régulièrement posés depuis et qu'ils se posent désormais d'eux-mêmes, dans le vif du sujet. Et cela ne relève certes pas du hasard que, déjà présents au sommaire de Onze analyses, les Nicole Brossard, Normand de Bellefeuille, Louise Cotnoir, Joseph Bonenfant, Lise Guèvremont, Hugues Corriveau aient par la suite présidé à la fabrique et à la conduite éditoriale de la revue ou qu'ils en soient demeurés, avec tant d'autres, des lecteurs, scripteurs ou animateurs conséquents.

Bref, dix ans sont passés; la BJ est devenue «nouvelle», cent numéros ont été publiés. Et au moment où je m'apprête au survol de quelques «temps forts» d'une décennie de lectures BJ/NBJ, cette digression liminaire aura voulu rappeler le contexte de ce «premier temps» d'une effervescence intellectuelle, lequel fut pour nombre d'entre nous embrayeur d'un engagement de lecture. D'écriture. De lecture-écriture. Voilà. Relisons: «La Nouvelle Écriture" Colloque NBJ 1980 (no 90-91), Poésie 1980 (no 92-93), Poésie 1981 (no 100-101), Écritures 1983 (no 122-123), Traces écriture de Nicole Brossard (no 118-119) et Intellectuel/le en 1984? (no 130-131).

«Ce dont je parle est ce que j'écris - Ce que j'écris «me»» parle. (Claude Beausoleil)

\section{Penser l'écriture, écrire la pensée}

Inscrire l'actualité de la recherche scripturale, stimuler voire provoquer la fabrique expérimentale du texte comme instance de différenciation du sens et comme pratique de l'«autrement dit»: voilà un projet, souventes fois rappelé, certes à travers des variables théoriques, dans nombre d'énoncés de politique éditoriale et par les diverses équipes, mutantes, qui ont assumé la responsabilité de la BJ/NBJ. Par ailleurs, cette constance fondamentale dans la visée - dans l'angle de tir et dans l'exercice - s'est régulièrement discutée, révisée à la lumière des réflexions individuelles qui animaient des parcours singuliers d'écriture. Adviennent alors certains événements-bilans, certaines "situations» théoriques où l'écriture se pense, énonce les idées - fixes, fluctuantes - qu'elle entretient vis-à-vis d'elle-même. S'écrivant, l'écrit fait le point - marque ses points, discute ses "effets». Ainsi en est-il du colloque NBJ 1980, «La Nouvelle Écriture», dont les «actes» - le terme se prévaudrait ici de toute sa force de frappe - seront publiés dans le numéro 90-91, mai 1980 . A la vérité, rien de plus divers, de plus foisonnant, de plus «écrit» justement, que les interventions réunies ici, tant il est vrai que toute écriture nouvelle, et dans le moment même où elle s'informe de sa nouveauté et de sa différence, ne saurait être que plurielle, éclatée, stratégiquement inachevée: adonnée, 
toute, à une intertextualité (dé)concertante, polydirectionnelle. Rendre compte alors de la mobilité d'une constellation? Seule la neutralité statistique d'un glossaire, alignant les plus hautes fréquences conceptuelles et sémantiques, pourrait.témoigner de la polyphonie des axes et des accents névralgiques, en même temps qu'il décèlerait, dans les récurrences et les echos, les indices toujours provisoires d'une permanence ou les pistes friables d'une unité. Autour de la question - capitale - du texte, Nicole Brossard a déjà établi un sommaire succinct des principales occurrences thématiques: «car le texte, (...) «résume»; il se résume autour de quelques mots: ville, sexe, texte, corps, désir, graphie, oeil (celui du cinéma, celui de la photo). Le texte est idéogramme 2 \%.

De même, Roger Des Roches rappelant les exigences «nouvelles» de la «nouvelle écriture», en a schématisé les forces nécessaires: «Il y avait les grands thèmes: le texte: la matérialité de cet instrument (...), le sexe: parce qu'il y avait d'étonnantes remises en question (...), les différences dans tous leurs états (...), donc les politiques dans tous leurs états ${ }^{3}$ ").

Prenons donc appui sur la coïncidence de ces synthèses et voyons comment quelques-uns de leurs termes-clefs se disséminent et se parlent à travers d'autres voix.

Écrire:

VILLE: «Remontant le long d'une mémoire enferrée, le corps accroché à ses agglomérations, la ville surgissait de partout. Ville itinéraire que j'arpente ${ }^{4} \gg$.

(et)

SEXE: «le tranchant maintenant il est là près du devenir et de sa géographie on le respire dans une forme toujours instable le ciel le sexe à vous de choisir ${ }^{5}$ \%.

TEXTE: «Un TEXTE m'entreprend 6 . «La nouvelle écriture parle le texte dans le texte ${ }^{7}$.

CORPS: «à rafistoler ces corps, ils ne signifient plus rien, dans l'abondance. Craindre ces boursouflures de vie, puissances en démesures, cancers en bonne santé ${ }^{8}$ 》.

DESIR: «J'hésite et pourtant j'écris et c'est peut-être ce que je veux écrire. Des indices qui accompagnent la raison du désir d'écrire 9".

Mais par dela ces accointances ou ces proximités dans la nomination des motifs, à la fois choisis et délibérément obsessionnels, s'avouent, dans la quête même des «différences», d'autres affinités électives: tout d'abord, on veut que la diction même de ces thèmes s'opère tour à tour dans l'acte de 
la jouissance - ("une mise en acte de la jouissance ${ }^{10}$ ") - et dans «l'activité de la forme ${ }^{11}$ ». Car il s'agit toujours d' «écrire l'activité du sujet de l'écriture ${ }^{12}$ 》 et, par conséquent, d'accuser la présence et la conscience du «je», non plus écran mais écrivant ("vécrivant»): «la modernité est l'épreuve ultime que je dois subir pour continuer à être ${ }^{13^{\prime}}$. De l'écriture, comme acte informé - comme forme-sens jouie et jouée par un je -, à la jouissance, découle une autre consigne, une autre connivence: «ne plus refouler la passion et la pensée la passion de la pensée ${ }^{14}$ ), laquelle s'énonce, à maintes reprises, en des formules analogiques. Significatives coïncidences, que je souligne ici:

(...) une forme de recueillement et de concentration que j'appelle la pensée de l'émotion et l'émotion de la pensée 15.

Chronophotographie. Je lis Philippe Haeck:

Je pense que penser est une chose difficile. (...) Apprendre à penser c'est chercher un maître et une sage-femme qui nous montreront à respirer, d̀ mêler amour et intelligence ${ }^{16}$.

et Claude Beausoleil:

Ainsi la passion de l'ecriture n'est pas une accumulation d'effets, elle est un terrain d'investissement entre le sens et le soi ${ }^{17}$.

et France Théoret:

Parlant comme je l'ai fait, j'ai oublié ce qui me sollicite le plus, et cela quotidiennement, la pensée et l'écriture sensuelles ${ }^{18}$.

Ainsi, on aura mésestimé les intentions de la nouvelle écriture en affirmant qu'elle postulait, narcissique ou miroir d'elle-même, ces formes mécanistes vides de sens ou d'émotion; tout se passe plutôt comme si elle prenait à son compte et réactivait sans cesse l'assertion lucide de Henri Meschonnic:

«Parler du langage est nécessairement passionnel».

Cette passion, elle l'assume dans la «résistance» (Hugues Corriveau) aux normes et aux formes de la «pensée conforme» (Marie-Claire Vaillancourt); elle la traduit, dans cette pratique systématique de l'uindécidable» et de l' "excès", par la fusion entre "texte - écriture - poème poésie - théorie - critique» comme entreprise conjuguée de totalisation (cf. l'hologramme magnétique chez Yolande Villemaire), de mixte et de transgression; elle la proclame dans la remise en cause du «référentiel» et de l'«intention lisible» (Normand de Bellefeuille) ou encore dans son 
indiscipline à l'égard des pouvoirs du père (François Charron, Philippe Haeck, Francine Saillant) et des censures portées par les discours d'autorités et d'ordres établis - tant il est vrai qu'entre auteur et autorité ... - ; enfin, elle l'inscrit, souvent en une patiente écriture spiroïdale, explorant tour à tour la volute et la saccade (en est exemplaire, ici, l'admirable dérive textuelle d'un François Charron: "L'Écriture commence par un rêve»), le cercle ou la marge. Écriture et passion: écriture passionnelle; elle se réclame d'une pensée en acte et d'un acte de langage. Portée entre l'état d'urgence et la ligne du risque.

Textes et tests:

Poésie 1980, Poésie 1981, Écritures 1983.

Dès Poésie 1980, qui engagera Poésie 1981 puis Écritures 1983, la formule de l'anthologie annuelle m'apparaissait comme un instrument précieux à la fois de rétrospective et de prospective des pratiques d'écritures. En effet, avec la diversité d'un ouvrage collectif, étaient données comme par condensation, réfraction et en avant-première de lecture, les tendances ou les postures des livres de l'année à venir et ainsi, les lignes virtuelles ou les possibles des parcours d'écritures. Car l'intérêt premier de ces numéros «répertoires» réside, comme l'indique Michel Gay dans son «liminaire» à Poésie 1981, en ce qu'ils corrigent des retards: «le recueil de poèmes, s'il lui arrive d'être publié, est lu avec des retards plus ou moins considérables. Ainsi se perd de vue le moment précis où le poème aurait dâ s'inscrire. C'est cette sorte de retards que le présent numéro voudrait contribuer, entre autres, à combler ${ }^{19}$,. Ce faisant, ces anthologies atténuent la distance entre l'avènement des livres sur le marché et l'actualité ou le moment de leur écriture. En ce sens, les textes «préalables» qui y paraissent s'éprouvent à la fois comme des «tests» à la lecture et ils fournissent des indices quant aux champs scripturaires qui sont investis à une période donnée.

Certes la ponctualité et l'arbitraire inévitable de ces "collections» appellent des prudences de synthèse et sans doute faut-il se prémunir ici de la remarque de Barrès: "Vous sortez des anthologies et vous jugez tout d'après elles!» Ainsi, n'est-il pas possible en ces lignes de faire le point sur les grandes incidences thématiques et les constances ou les expérimentations formelles qui caractériseraient plus de trois années d'écritures chez plus d'une trentaine d'écrivains (rétrospectivement 35 auteurs en 1980, 29 en $1981 ; 41$ en 1983). À elle seule, par exemple, l'observation du minimalisme progressif d'un Jacques Brault (de «Cinq solitudes» (1980) a «Timbres postes» (1982)) ou du passage, chez Lucien Francoeur, de la réduction hiéroglyphique ("L'affichage sauvage» (1981)) à un lyrisme néo-nelliganien («Nelligan rêve rock» (1983)), appellerait des commentaires minutieux et mériterait 'd'être confrontée à l'itinéraire plus complet des auvres. De même l'èstimation des critères - compte tenu de la sollicitation éditoriale et de la décision des répondants - qui président à la constitution des corpus et motivent la présence ou l'absence de tel auteur, réclamerait soit l'analyse 
des rythmes individuels de la production des textes ou encore celle des relations de lecture-écriture que les intervenants entretiennent avec la revue 20 .

Cependant, en dépit de la diversité des produits - diversité dans une continuité qui sans cesse se révise et se questionne -, ici encore se posent des correspondances de points de vues, $s$ 'indiquent des lieux d'exploration. Ainsi, dans «Le Métier et les pièges», Roger Des Roches rappelait la volontaire primauté de l'affirmation du JE dans le texte:

(...) nous avons décidé - oui, ça s'est imposé

- que le JE reprenait une place dans le texte (notez bien que le JE ne reprenait pas sa place dans le texte: il prend UNE place - parce qu'il n'y était plus - il se cherche une place), nous avons décidé qu'il serait intéressant et nécessaire de faire des textes sur la tension et sur le sentiment, avec une autre sorte de langage (c'est toujours une autre forme de langage; il ne faut jamais que ça se donne, jamais!), et il fallait voir ce que JE pourrait y accomplir. Il serait intéressant de travailler sur l'intime, sur l'intimisme, sur l'illustration de «sujets» plutôt que d'sobjets $21_{\text {». }}$

La même année, dans sa présentation de l'anthologie Poésie 1980, Michel Beaulieu, sur un ton sans doute plus polémique, abondera dans un sens analogue:

Plusieurs signes avant-coureurs permettaient de prévoir un retour en force du je, du lyrisme, du lisible, d'un vocabulaire que certains n'avaient pourant, malgré les discours dominants qui se sont succédés depuis 10 ans, volontiers terroristes, jamais abandonnés au cours de ces années 22 .

Faudrait-il entendre en ces propos une visée rétroactive en règard de l' «expression» ou du ré-investissement du référent subjectif, intimiste, dans le travail textuel? Ou encore y lire des dissidences par rapport à l'खintention lisible» déniée en telles interventions théoriques énoncées dans le même temps? Il me semble plutôt que la panoplie des textes publiés dans les trois anthologies tend à proposer, dans le procè's d'écriture, des conciliations 23 : entre émotion (toujours «la pensée de l'émotion ...») et implication (aussi, «les activités de la forme»), entre référence et différence, entre spontanéité expressive (cet exercice de la dérive) et travail: la revue demeure un lieu de «travail». Près de la langue, ce palais du poème ${ }^{24}$, est-il confirmé dans la livraison de Poésie 1981. Certes, dans sa pratique concrète, au moment où elle met en place (en texte) les thèmes et les formes qu'elle s'est déjà reconnus, la nouvelle écriture se veut labile et exploratoire; en même temps 
qu'elle se livre aux appels du texte qu'elle engendre, elle se commet résolument dans les règles de l'infraction, de la dépense et de l'inflation

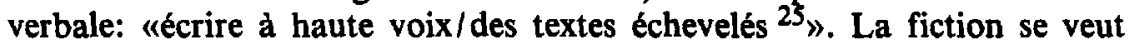
friction entre le réel et l'imaginaire: explosif, le fragment se thématise et s'inscrit en sa dispersion, comme ensemble et réseau ${ }^{26}$; enfin tout texte, comme émergence d'un lieu zéro de l'écriture, conspire à une patiente et ambitieuse intertextualité:
Ainsi poursuivre le travail millénaire entreprendre la tournée des grandes énigmes et terminer les textes jamais commencés ${ }^{27}$.

En bref, cette écriture rappelle sans cesse que de l'auto-analyse de son projet (de son procès) au travail de son inscription, son enjeu s'effectue constamment entre le machinisme et l'anamorphose, entre l'influence connivente et la différence marquée: aussi, entre le «je» (comme jet textuel) et les objets de sa fabrication.

«La forme vise la destruction du sens. Le sens livre sa formule malgré la ratures. (France Theoret)

J'ai évoqué comment certains d'entre nous avions été subjugués et aussi nouvellement questionnés et informés par l'enseignement d'une Hélène Cixous. Sans doute, alors, n'étions-nous pas encore pleinement conscients que le «grand sur-moi français» conditionnait ou supervisait, pour une part, notre relation à l'écrit. Fait singulier, dix ans plus tard, en une formule où il faut entendre la part du «plaisir courtois», Normand de Bellefeuille imputera à Nicole Brossard une responsabilité analogue de tutelle ou d'emprise sur les écritures d'ici:

Pourtant, il nous faut bien le voir: ce que Nicole Brossard a déjà, très justement d'ailleurs, identifié comme le «grand sur-moi français» planant sur l'écriture d'ici a été relayé, depuis quelques années, par un non moins «grand sur-moi Brossard"; étonnant tout de mềme pour une écriture dont la modernité avait précisément pris assise sur une discrétion du sujet ${ }^{28}$.

S'il ne m'appartient pas d'interroger maintenant et dans le détail ce que telle pratique d'écriture actuelle doit au texte brossardien, il reste néanmoins que 
cette ouvre capitale a souvent été perçue comme «sémaphore» textuel et que, de fait, elle a laissé des "traces», par aimantation ou irradiation, dans nombre de livres ${ }^{29}$. D'ailleurs les séquelles ou les indices de complicité engagés par les travaux de Nicole Brossard s'avouent très clairement dans les commentaires analytiques énoncés lors du colloque qui lui est consacré, et ce, dans la tonalité même des écritures qui s'y impliquent; en effet, cellesci se jouent constamment dans une sorte d'osmose, ou de réflexion spéculaire ou de mimétisme ludique: la re-lecture se dédouble, se fait récriture: au second degré. Le discours critique ouvre l'œuvre (rature son e muet, pourrait-on dire ...) pour s'y activer et, en un sens, s'y mettre en abyme. Cette attitude de stimulation intertextuelle est d'autant plus dynamique et indicative d'une forte solidarité à l'égard du texte brossardien que plus d'un exégète aura déjà noté à la fois la «neutralité» et l'implicite «singularité» de celui-ci.

Ainsi, alors que France Théoret écrit: «Le texte travaille à s'effacer. (...) Le texte cherche absolument la neutralité. (...) Nicole Brossard cherche à faire dire le moins possible. (...) Le langage s'énonce sans sujet d'énonciation dans un présent délivré de la pesanteur mais il n'arrive jamais à la formulation tautologique ${ }^{30}{ },-$, André Roy indique différemment la version de sa lecture:
Créatrice d'une nouvelle langue - on reconnaît du Brossard au premier coup d'oeil -, elle témoigne aussi de la pratique de l'écriture comme subjectivité qui pulvérise toute tentative de sédimentation d'une identité, et ce, dans la pluralisation des sens en nouant symbolique et désir ${ }^{31}$.

Plus qu'une opposition, me semble s'indiquer ici une tension et se rendre visible la figure de l'oxymoron qui, avec celles de la métonymie et de la synecdoque ( $c f .:$ La partie pour le tout), articulent la poétique de Nicole Brossard, en accusent le relief rhétorique. Tension, donc, entre «invariance» et "unicité» subjective, entre totalité concentrique («centre blanc») et pluralité expansive («mécanique jongleuse»). On comprendra alors que la critique n'a pas d'autre choix que celui de s'immiscer dans cette concentration, non pas tant pour la dénouer ou en juguler les énergies, mais plutôt pour faire partie, par le biais d'un discours contrapuntique, de ce projet alternatif de réduction et de totalisation. Adviennent ainsi des lectures qui posent sur l'œuvre ces: «Regards, Reflets, Reflux, Réflexions ...» (Louise Forsyth), des «lectures intimes» (Michèle Saucier) ou cette "lecture tangentielle» (Louise Cotnoir); toutes, de celle, euphorique, de Yolande Villemaire ("Vénus est une jeune femme rouge toujours plus belle») qui parle sa propre fiction, à la «Fieffée désirante» de Louky Bersianik, qui indique l'apport percutant de l'œuvre de Brossard pour l'inclusion de la parole des femmes «dans le sens», s'élaborent sur le mode du palimpseste réverbérant. Comme si le texte brossardien ne se laissait pas dire autrement qu'il ne se dit lui-même mais agissait plutôt comme 
embrayeur d'écritures plurielles, polyphoniques. Comme cet hologramme que Louky Bersianik décrit ainsi: «Image fixe qui exclut l'idée d'un centre, et qui succède à l'image mobile de la spirale, celle-ci prenant naissance en son centre et évoluant vers l'excentricité ${ }^{32}$ ». Voila, me semble-t-il, la représentation figurative (la "picture theory») de toute écriture qui s'entend à "cerner» le texte brossardien, à «faire le tour» du "centre blanc»: circonvolutions, pourtours, pivots, gonds, spires, cycles, autant d'images d'une célérité ronde (d'un vertige, justement) qui oscille entre l'inclusion et l'évolution et que Pierre Nepveu, en une solide microlecture, a condensé par une vocable unique: «Je voudrais ici parler d'un seul mot: énergie ${ }^{33}$ ". Traces d'écriture: là où la dépense induit la synthèse. Et vice versa.

$+$

*

\section{Penser la pensée: Intellectuel/e en 1984?}

1984. Si cette année-fétiche inclinait à l'augure ou à la prémonition, au constat ou au bilan, elle aura plutôt incité la NBJ à la «question»: Intellectuel/le en 1984? Depuis ces années 70 que j'évoquais tout au début, quelques-uns d'entre nous n'occupent plus exactement la même place sur l'échiquier de la salle de classe. Professeurs au collégial, chargés de cours à la pige, postes rarissimes dans les universités ${ }^{34}$. Donc, «profs». dans la trentaine: certes, ce n'est pas vieux, ce n'est plus jeune! Entre l'ère du soupçon et la force de l'âge ... Et intellectuel/les? Ce point d'interrogation sera circonstancié:
C'est au cours du conflit opposant le gouverne- ment québécois et les enseignants/tes que nous est venue l'idée de rassembler des textes sur le rôle de l'intellectuel/le au Québec. (...) Toutes les principales avenues de l'activité intellectuelle québécoise y sont explorées: la question nationale, le militantisme de gauche, la critique, l'écriture, le féminisme, la philoso- phie, etc. ${ }^{35}$.

Certes, faut-il le rappeler, après ces «événements de l'hiver 1983» sanctionnés par une loi 111 que d'aucuns, forçant à peine les mots, qualifieront de "notre Loi des mesures de guerre en Education ${ }^{36}$ ", je crois bien que nous étions devenus aphones. L'impact des décrets nous laissait bouche bée, stupéfaits. En ce sens, l'initiative prise par Normand de Bellefeuille et Louise Dupré de rassembler des textes rétorsifs d'intellectuels concernés, forçait la réflexion et nous rendait l'«âge de la parole» (sans doute que nous revenait du même coup en mémoire la rage urgente de ce poème fameux de Roland Giguère - «la main du bourreau finit toujours par pourrir» - dont nous avions procédé, jadis, à une structurale dissection). Fait à noter cependant: si le «prétexte» des textes qui se donnent ici s'inscrit dans la perspective d'une dénonciation des «événements», 
quelques-uns d'entre eux seulement les contestent de front ou avec cette violence ou cette force de frappe stratégique que réclamait, dans les circonstances, le rétablissement légitime de la loi du talion ${ }^{37}$. Tout se passe plutôt comme si, heurtés à vif dans leur droit d'intervention, les intellectuels avaient préféré passer outre à l'indigente conspiration politicienne portée contre eux, pour recouvrer en marge des événements et des tactiques d'intimidation, dans l'apparente digression de discours qui sondent davantage les racines et les causes des abus de pouvoir que leurs vénales manifestations, la liberté même de leur pensée. Cela donne des essais alertés et mis au point qui, par une juste voie d'évitement, se font aux idées en exhibant la viduité des lieux où «ça» n'a pas pensé; tant il est vrai qu'en regard d'une actualité qui délire ou des intrigues de petits contremaîtres d'occasion, «l'intelligence ne peut rien: voilà ce qui fait après coup (...) sa force ${ }^{38}$ ").

$\mathrm{Si}$ les réponses (souvent elles-mèmes interrogatives) à la question soulevée dans ce numéro, constituent, dans l'ensemble, un remarquable panorama des paramètres polémiques qui s'activent en divers lieux de la pensée québécoise, deux textes ont, plus particulièrement, engagé ma complicité.

Ainsi, il faudra relire, de Normand de Bellefeuille: «Théorie: la Pensée en panne, réinventer la vitesse ...» Alerte, le réquisitoire dénonce toutes les modes idéologiques, «théories de l'«aura» et délire zodiacal, énergies diverses (l'énergie comme «relève» à l'idéologie) et gouroumanie galopante, égyptophilie, néo-mysticismes, etc. ${ }^{39}$, y compris celle de la tolérance sympathique, qui agitent, par vagues fluctuantes, ce qu'il est parfois convenu d'appeler «le milieu». (D'ailleurs, ce texte, comme s'il constituait le blason percutant du numéro, sera lu tel un embrayeur polémique, une cible; dans «le milieu», justement). Certes, le ton est "armén; mais quelle santé intellectuelle s'avoue dans cet affat de la dissidence vigilante quand, se refusant à toutes les habitudes comme à tous les courants, la pensée tient front. Plus intimiste et confident, André Lamarre dans " Journal d'une taupe, juillet 1983" propose, en une sorte d'autobiographie intellectuelle, la chronique d'une époque, d'une génération: celle des trente ans. S'y glissent, en une voix où se fondent dérision et lucide nostalgie, nos engouements culturels et nos dépits politiques, nos défections et nos déceptions sociales, nos valeurs et nos engagements revus et corrigés. «Partir de là. Creuser ${ }^{40}$ 》 Réalisme et cynisme d'un portrait-robot: entre la recherche du temps perdu et le choc du futur.

«Intellectuel/le en 1984?» Les réponses tour à tour passent aux aveux, aux actes de la pensée. Ecrire encore: des «actes retrouvés». 


\section{Exit}

Quelques «accents aigus» ont été indiqués, marqués certains «temps forts» d'un lieu de travail ou d'un laboratoire de langage qui, depuis 20 ans, à la fois provoque et convoque l'écriture. Il s'est agi ici d'une lecture $T$ donc d'un choix - et, par conséquent, d'une lecture lacunaire. Au hasard, je retire encore quelques titres de mes rayons: $A$ quelques obsessions près (NBJ, no 77), Science-fiction (no 79-80), Le Fantastique (no 89), La Femme et la Ville (no 102, L'Infratexte (no 103), La Bande dessinée (no 110-111), La Complicité (no 112), Femmes (no 124), Ce qui les séduit (no 127-128), OuLiPo Qc (no 134), Révélatrices femmes et photos (no 136-137); tous ces livres, et ce même du strict point de vue titulaire, témoignent d'une actualité diverse, d'une proximité constante avec les questions capitales qui stimulent la littérature contemporaine. Certes de la «barre» à la «nouvelle barre du jour", une bibliothèque s'est constituée. J'aurais voulu tout revoir de ce qui est déjà paru alors même que je suis sollicité par ce qui paraît. Car la nouvelle écriture qui a cours ici continue d'inscrire - selon le mot que j'emprunte à Rosenberg qui définissait ainsi la modernité - la «tradition du renouveau»; et c'est bien parce que cette écriture perdure en sa nouveauté, que persiste à la lecture, comme à la re-lecture, le plaisir.

1. Nicöle Brossard, «Vague de précision», BJ, Onze analyses, no 39-41, printemps-été 1973, p. 3.

2. Nicole Brossard, «L'épreuve de la modernité», NBJ, Colloque NBJ 1980, «La Nouvelle Écriture», no 90-91, mai 1980 , p. 62.

3. Rogers Des Roches. «Le métier et les pièges», ibid., p. 157.

4. André Beaudet, "L'état de veille» (èxtrait), ibid., p. 107.

5. François Charron, «L'écriture commence par un rêve», ibid., p. 16.

6. Claude Beausoleil, «Quelques désordres suivi de Son image et la rupture», ibid., p. 118.

7. France Théoret, "L'implicite et l'explicite de la nouvelle écriture», ibid., p. 166.

8. Francine Saillánt, «Ā savoir ouvrir par hasard», ibid., p. 138.

9. Claude Beausoleil, «Lè désir d'écrire», ibid., p. 111.

10. François Charron, «L'écriture commence par un rêve», ibid., p. 18.

11. Ibid., p. 30.

12. Ibid., p. 26 .

13. Nicole Brossard, «L'épreuve de la modernité», ibid., p. 57. 
14. François Charron, ibid., p. 29.

15. Nicole Brossard, ibid., p. 67.

16. Philippe Haeck, «De la curiosite», ibid., p. 77.

17. Claude Beausoleil, "Le désir d'écrire», ibid., p. 114.

18. France Théoret, «L'implicite et l'explicite de la nouv̧elle écriture», ibid., p. 170.

19. Michel Gay, «Liminaire», Poésie 1981; NBJ, no 100-101, mars 1981, p. 7. Il importe, en effet, d'observer comment nombre de textes ou de, gragments donnés dans Poésie 1981 seront repris dans des livres qui paraîtront entre 1983 et 1984, accusant ainsi un retard de plus de 2 ans par rapport a la revue. Ainsi, et entre autres, "Pop Cycle» de Claude Beausoleil sera repris dans Une certaine fin de siècle (Noroît, 1983), "Travaux de sape» de Hughes Corriveau dans Revoir le rouge (VLB, 1983), "Mutineries» de Louise Cotnoir dans Plusieures (Éditions du remue-ménage, 1984). «Le livre du devoir (extrait)» dans Le livre du devoir (Herbes rouges, 1983), «Programme double» de Louis Dupré dans la Peau familière (Éditions du remue-ménage, 1983).

En outre, il serait intéressant de voir; s'il y a lieu, le travail de «rature» ou de «version» qui s'effectue à l'intérieur des textes, d'un' état de publication à un autre. Quelques variantes que j'ai observées entre le «fragmenț et le «livre» de Normand de Bellefeuille sont extrêmement révélatrices du dispositif textuel et pourraient conduire à une fructueuse analyse comparative.

20. Ainsi; par exemple, si les noms de François Charron et de Normand de Bellefeuille apparaissent aux sommaires des anthologies de 1980 et 1981 , ils ne se retrouveront pas a celui de Ecritures 1983. Pour sa part, Madeleine Gagnon ne sera présente que dans cette dernière livraison où elle donne, par ailleurs, un texte important: «L'étrange figure du lent destin des choses» (p. 129 et ss.).

21. Roger Des Roches, Colloque NBJ, «La Nouvelle Ecriture», p.159.

22. Michel Beaulieu, "La poésie en 1980", NBJ, Poésie 1980, no 92-93, juin 1980, p. 8-9.

23. S'y affirme aussi une progressive extension vis-à-vis de la définition du texte. En ce sens, alors que Michel Gay écrivait dans son «liminaire» a Poésie 1981: «LA NOUVELLE BARRE DU JOUR n'est pas exclusivement une revue de poésie. Mais la plupart des textes qui y circulent (...) sont marqués du coin du langage et de la pensée poétiques» (p. 7), on aura observé que, glissement significatif, l'anthologie de 1983 se présentera sous le titre: Ecritures 1983.

24. Michel Gay, ibid., p. 7.

25. Claude Beausoleil, «Six textes», Poésie 1980, p. 26.

26. Voir à ce propos: Paul Chanel Malenfant, «La poésie cette année: de l'écart et de l'éclat", Livres et Auteurs québécois 1980, PUL, Québec, p. 81-84.

27. Lucien Francoeur, "L'affichage sauvage», Poésie 1981, p. 82.

28. Normand de Bellefeuille, «Suite logique, Pour une grammaire de la différence», NBJ, Traces écriture de Nicole Brossard, no 118-119, novembre 1982, p. 97.

29. Louis Dupré le confirme dans "Les utopies du réel»: "Que Nicole Brossard soit une auteure moderne, qu'on lui attribue l'introduction, au Québec, de la nouvelle écriture, voilà qui semble évident en 1982". Ibid., p. 87.

30. France Theoret, «Le sens de la formule», Traces ecriture de Nicole Brossard, p. 80. De même, Normand de Bellefeuille a observé, chez Nicole Brossard, cette prédilection marquée, et ce du point de vue grammatical meme, pour le uneutre» et l'«invariable».

31. André Roy, "uLa verge au beau tarif»; la différenciation signifiante généralisée», ibid., p. 114.

32. Louky Bersianik, «Fieffée désirante», Traces, p. 110. Nicole Brossard elle-même a indiqué le fonctionnement de ce dispositif: «(...) il ne faut pas oublier la notion d'hologramme qui déplacée au niveau de l'écriture m'incite à vouloir faire le tour d'un mot, d'une idée, d'un concept afin d'en saisir toutes les dimensions. De même qu'il s'agit de faire le tour de ma propre subjectivité». «Entretien avec Nicole Brossard sur Picture Theory", ibid., p. 178. 


\section{DOSSIER 151}

33. Pierre Nepveu, «Nicole Brossard: Notes sur une écologie», ibid., p. 139.

34. Sans compter les autres, tous les autres pour qui la «question» mème tourne à vide: "Que fait-on, aujourd'hui, lorsqu'on appartient à cette génération d'intellectuels arrivés «un peu trop tard» ou «au mauvais moment» et que l'on se retrouve exclus de ce qu'on appelle la «population active»? À quoi peut-on s'attendre (comme encouragement ou solidarité) ou comment intervenir autrement tout en faisant bon usage de sa formation?" (Sylvie Gagné, «L'exil des intellectué(e)s», Intellectuel/le en 19847 NBJ, no 130-131, octobre 1983, p. 9.

35. Normand de Bellefeuille, «Liminaire», ibid., p. 5.

36. Robert Hébert, «Hypothèses laconiques sur un lieu en temps de paix», ibid., p. 114.

37. Plus “immédiatement» révoltés et vindicatifs me semblent les textes de Francine Saillant, Robert Hébert et Louise Dupré.

38. Robert Hébert, «Hypothèses laconiques ...», ibid., p. 121.

39. Normand de Bellefeuille, "Théorie...», ibid., p. 48.

40. André Lamarre, «Journal...», ibid., p. 99. 